\title{
Biodegradable 3D Printed Scaffolds of Modified Poly(trimethylene carbonate) Composite Materials with Poly(L-lactic acid) and Hydroxyapatite for Bone Regeneration
}

\author{
Honglei Kang ${ }^{1}$, Jiangxu Dong ${ }^{1}$, Zhi Wei Liu ${ }^{1}$, Shengmin Zhang ${ }^{2}$, Fan Liu ${ }^{1}$, Feng Li ${ }^{2}$, and \\ guoping yan $^{1}$ \\ ${ }^{1}$ Wuhan Institute of Technology \\ ${ }^{2}$ Huazhong University of Science and Technology
}

September 25, 2021

\begin{abstract}
The biodegradable scaffolds based on biomedical polymeric materials have attracted the wide interest in bone transplantation for clinical treatment to bone defects without the second operation. The composite materials of poly(trimethylene carbonate), poly(L-lactic acid) and hydroxyapatite (PTMC/PLA/HA) were prepared by the modification and blending of poly(trimethylene carbonate) (PTMC) with poly(L-lactic acid) (PLA) and hydroxyapatite nanoparticles (HA). The PTMC/PLA/HA scaffolds were further prepared by additive manufacturing using the biological 3D printing method. The PTMC/PLA/HA composite materials and scaffolds were also characterized by fourier transform infrared spectroscopy (FT-IR), GPC, automatic contact-angle, SEM, XRD, differential scanning calorimetry (DSC) and thermogravimetry (TG). Subsequently, their properties, such as the mechanic, biodegradation, drug controlled release, cell cytotoxicity, cell compatibility in vitro and proliferation/differentiation assay in vivo, were also investigated. Experiment results indicated that PTMC/PLA/HA scaffolds possessed the low toxicity, good biodegradability and good biocompatibility and then improved the cell proliferation of osteoblast cells (MC3T3-E1). Moreover, PTMC/PLA/HA enhanced the adhesion and proliferation of MC3T3-E1 cells, allowing penetration of host cells within the construct boundaries, and then can enable the growing of bone cells and induction of bone tissue regeneration. Therefore, PTMC/PLA/HA composite materials can be used as the potential biodegradable scaffolds for bone regeneration and tissue engineering in vivo.
\end{abstract}

\section{Introduction}

The bone transplantation is often used in clinical treatment to bone defects. Currently the demand for bone transplantation has increased significantly in the world. The autogenous bone transplantation is currently adopted in surgical operation, however, there is some disadvantages, such as lack of source, difficult to repair large area of bone defect and some postoperative complications. Therefore, the development of novel biodegradable scaffolds based on biomedical polymeric materials for bone transplantation has become the current research difficulties and hot spots ${ }^{1-8}$.

Benefiting from the porous and network structure provided by the biodegradable polymeric scaffolds, cells in the bone marrow could attach onto them and repaired the bone defect with out a second surgery. It was reported that biodegradable aliphatic polyesters including the poly( $\varepsilon$-caprolactone) (PCL), poly(L-lactic acid) (PLA) and poly(trimethyl carbonate) (PTMC) revealed a good biological ability at bone defect reparation and drug delivery system ${ }^{9-13}$. PTMC is a kind of biomedical polymer, which possesses the low toxicity, good biocompatibility, good biodegradability, good flexibility, and good surface corrosion properties, and will not produce strong acid compounds after degradation. Meanwhile, PTMC appears as an elastic polymer at body temperature. So it has a good application prospect in the tissue engineering, drug controlled release and 
other biomedical fields. Moreover, PTMC is one preferred synthetic thermoplastic polymeric biomaterial which can be used in 3D printing and suitable to manufacture the biodegradable scaffold and biofabrication for tissue regeneration and tissue engineering. However, the poor mechanical property of PTMC generally hampered its wide application. Thus PTMC was modified to prepare the composite materials using the copolymerization and blending methods by some polymers and inorganic nanomaterials ${ }^{14-16}$.

Poly(L-lactic acid) (PLA) is also a biodegradable synthetic polymer and possesses some advantages, such as low toxicity, good biocompatibility, good biodegradability, good mechanical and physical property. It can be easily processed in many ways, such as 3D printing, extrusion, spinning, biaxial drawing and injection blow molding. However, PLA appears the hard, crisp character, bulk degradability and acidic degradation products, which inducing to local $\mathrm{pH}$ reduction and some adverse inflammatory reaction. So some modification methods are used to improve remarkably its properties including hydrophilicity, degradation and flexibility ${ }^{17-19}$. It is interesting that the properties of PLA and PTMC present good complementarity and then PLA is a good choice to modify PTMC.

The polymer composites with nanoceramics reflected a higher mechanical capabilities than the pure polymers due to their distribution of nanoparticles within polymer matrix which providing adequate strength and stiffness. Before the new tissue repaired the implant which is gradually degrading, the scaffolds could sustain a certain grade of physical stress ${ }^{20,21}$. Hydroxyapatite (HA) is one of natural mineralization form of the calcium apatite, which is the crucial component of teeth and skeleton. Due to its superiorities of excellent biological capability, low toxicity and conveniently to product, HA is widely applied in the bone and teeth implant operation. Moreover, the HA modified materials such as PLA could enhance its physical and biological properties by suppressing the self-acceleration of acidolysis. Meanwhile because of the alkalescent HA nanoparticles, the degradation process of ester bonds is also decreased. ${ }^{22-24}$.

The composite materials of PTMC, PLA and hydroxyapatite (PTMC/PLA/HA) were prepared by the modification and blending of PTMC with PLA and HA. Subsequently, the biodegradable PTMC/PLA/HA scaffolds were further produced by the additive manufacturing of PTMC/PLA/HA composite materials using the biological 3D printing method. Their propertiesin vitro and in vivo were also evaluated herein and expected to study its potential application in bone repair and replantation.

\section{Materials and methods}

\subsection{Chemicals}

All chemicals and solvents were of analytical grade. Tin (II) 2-ethylhexanoate $\left(\mathrm{Sn}(\mathrm{Oct})_{2}\right)$ was purchased from Sigma-Aldrich (Louis, MO, United States of America) and purified by redistillation in vacuo before use. Toluene and tetrahydrofuran (THF) were purified by redistillation over sodium. Triethylamine was refluxed under phthalic anhydride and dried over calcium hydride $\left(\mathrm{CaH}_{2}\right)$ before use. The growth medium was the Roswell Park Memorial Institute ( $\alpha$-MEM media: 10\% fetal bovine serum (Gibco. Co., United States of America), 100 units $/ \mathrm{mL}$ penicillium, $100 \mu \mathrm{g} / \mathrm{mL}$ streptomycin). Poly(trimethylene carbonate) (PTMC) and poly(L-lactic acid) (PLA) were synthesized by ring-opening bulk polymerization of trimethylene carbonate (TMC) and L-lactide, respectively, using tin (II) 2-ethylhexanoate as a catalyst ${ }^{25-27}$. PTMC was characterized by ${ }^{1} \mathrm{H}$ NMR (Varian, Inc. Corporate, Palo Alto, CA, USA), DSC (NETZSCH DSC 200 F3, Erich NETZSCH GmbH \& Co. Holding KG, Gebrüder-Netzsch-Strasse, Selb, Germany), automatic contact-angle measurement (SL200A/B/D Series, Solon Tech. Inc. Ltd., Shanghai, China) and gel permeation chromatography (GPC, Waters Corporation Milford, MA, USA). PTMC: ${ }^{1} \mathrm{H}$ NMR $\left(300 \mathrm{MHz}, \mathrm{CDCl}_{3}, \delta\right.$, ppm): $3.885\left(\mathrm{~m}, 4 \mathrm{H},-\mathrm{COO}-\mathbf{C H}_{\mathbf{2}}-\mathrm{CH}_{2}-\mathrm{CH}_{2}-\right), 2.035\left(\mathrm{~m}, 2 \mathrm{H},-\mathrm{CH}_{2}-\mathbf{C H}_{\mathbf{2}}-\mathrm{CH}_{2^{-}}\right)$. Glass transition temperature $(\mathrm{Tg})$ of PTMC is $-16.7^{\circ} \mathrm{C}$. The molecular weight $\left(\mathrm{M}_{\mathrm{n}}\right)$ was $3.1 \times 10^{5}$ and polydispersity was 1.45 . PLA: ${ }^{1} \mathrm{H}$ $\operatorname{NMR}\left(300 \mathrm{MHz}, \mathrm{CDCl}_{3}, \delta, \mathrm{ppm}\right): 5.2(\mathrm{~m}, 2 \mathrm{H}, \mathrm{C}-\mathrm{CH}-\mathrm{C}), 1.5\left(\mathrm{~d}, 6 \mathrm{H}, \mathrm{CH}_{3}\right)$. Glass transition temperature $(\mathrm{Tg})$ of PLA is $56^{\circ} \mathrm{C}$. The molecular weight $\left(\mathrm{M}_{\mathrm{n}}\right)$ was $1.9 \times 10^{5}$ and polydispersity was 1.52 . The osteoblast cells MC3T3-E1 were provided by the China Center for Type Culture Collection of Wuhan University, China, and were raised according to the method described in the literature ${ }^{28}$.

2.2 Preparation of poly(trimethylene carbonate), poly(L-lactic acid) and hydroxyapatite 


\section{(PTMC/PLA/HA) scaffolds}

The PTMC/PLA/HA and PTMC/HA composite materials were prepared by the blending of PTMC, HA or PLA. PTMC, PLA and HA (20 nm, purchased from Beijing Deke Daojin Science and Technology Co.) were added to dichloromethane and stirred to make them mixed evenly. Under a reduced pressure, the mixture was tardily evaporated to dry and then sheared into small pieces, subsequently they were dried in condition of vacuum for 48 hours to yield the composite materials.

The 3D printed PTMC/PLA/HA and PTMC/HA scaffolds (diameter $\times$ height: $4 \mathrm{~mm} \times 6 \mathrm{~mm}, 10 \mathrm{~mm} \times 2 \mathrm{~mm}$ ) were prepared using a Regenovo 3D Bio-Architect@ Pro Biological 3D printer (Hangzhou Regenovo Biotechnology, Ltd., Hnagzhou, Zhejiang, China) with PTMC/PLA/HA and PTMC/HA composites, respectively. Inner diameter of print head was [?] $0.41 \mathrm{~mm}$, extrusion pressure of print head was $0.3 \mathrm{MPa}$, and printing rate was $6 \mathrm{~mm} / \mathrm{s}$ at room temperature.

\subsection{In vitro degradation test}

The PTMC, PTMC/HA and PTMC/PLA/HA scaffolds (0.1g, diameterxheight: $10 \mathrm{mmx} 2 \mathrm{~mm})$ were suspended in $10 \mathrm{~mL}$ of PBS ( $\mathrm{pH}$ 7.4) in a sealed dialysis bag and then added $90 \mathrm{~mL}$ of PBS shaken in a 250 $\mathrm{mL}$ Erlenmeyer flask at $37^{\circ} \mathrm{C}$. The samples were taken out of the degradation medium at 1, 2, 3, 4, 5, 6 months, respectively.Rinsed with distilled water and then dried for $48 \mathrm{~h}$ in vacuo. The molecular weight and weight loss of scaffold at different degradation time were calculated, respectively.

\subsection{In vitro drug release study}

5-DOX-incorporated PTMC, PTMC/HA or PTMC/PLA/HA composite materials were obtained by blending doxorubicin (DOX, $10 \mathrm{mg}$ ) and PTMC, PTMC/HA or PTMC/PLA/HA composite materials (100 mg) in $20 \mathrm{~mL}$ THF and dried under vacuum. And then put them in a disc mold (diameterxheight: $10 \mathrm{mmx} 2 \mathrm{~mm}$ ) on a thermocompressor with loading pressure of $10 \mathrm{MPa}$, setting temperature of $110{ }^{\circ} \mathrm{C}$ and molding time of $10 \mathrm{~min}$.

The 5-DOX-incorporated PTMC, PTMC/HA or PTMC/PLA/HA scaffolds were suspended in $10 \mathrm{~mL}$ of PBS in a sealed dialysis bag and then shaken in $90 \mathrm{~mL}$ of PBS at $37^{\circ} \mathrm{C}$ in a $250 \mathrm{~mL}$ Erlenmeyer flask. A liqouts of the solution outside the dialysis membrane were replaced with same volume of PBS at various times intervals and tested at $256 \mathrm{~nm}$ by UV-Vis spectrophotometer (UV-2800 series, Unico, Shanghai, China). The changes of the concentrations of DOX were obtained from curves of the absorption $A$ versus concentration $C$ of DOX in PBS based on Lambert-Beer law.

\subsection{Cell cytotoxicity assay}

For cell cytotoxicity assay, cell counting kit 8 (CCK-8) assay was performed according to the manufacturer's protocol. Briefly, series of PTMC, PTMC/HA and PTMC/PLA/HA scaffolds were produced by 3D printing,parameters were set as following: thickness $=2 \mathrm{~mm}$, the diameter $=6 \mathrm{~mm}$, percentage of HA was set as $0 \%, 5 \%, 10 \%, 15 \%, 20 \%$ and $25 \%$, all scaffolds were rinsed in the distilled water and made dried, then they were sterilized by using ethylene oxide. MC3T3-E1 cells were counted and seeded on the surface of scaffolds in 48 -well culture plates at a density of $2 \times 10^{4}$ cells/well, cells were then cultured with complete $\alpha$-MEM medium for one and three days, subsequently CCK- 8 assay was conducted at each time point and tested at wave length of $450 \mathrm{~nm}$.

\subsection{Cell attachment and proliferation assay}

For cell attachment assay, two types of scaffolds formed with PTMC/HA and PTMC/PLA/HA were made by $3 \mathrm{D}$ printing, they were produced with a diameter of $6 \mathrm{~mm}$, thickness was designed as $2 \mathrm{~mm}$ and content of HA was set as $25 \%$. The scaffolds were precleaned and ethylene oxide sterilization was performed, MC3T3-E1 cells were then seeded onto the scaffolds in 24 -well culture plates at a density of $1 \times 10^{5}$ cells/well, cells were cultured with $\alpha$-MEM medium for 7 days, then rhodamine labeled phalloidin staining was performed. Briefly, cells were washed with PBS for one time and fixed with $4 \%$ paraformaldehyde for $15 \mathrm{~min}$, after washing 3 
times with PBS, phalloidin staining assay was added and incubated for 1 hour, after then 4',6-diamidino-2phenylindole (DAPI, Invitrogen, USA) staining was conducted for 15min. Subsequently, cells were washed for 3 times and images were captured with EVOS fluorescence microscope (Thermo Fisher Scientific).

For proliferation assay, cells were treated with 3-(4,5-dimethyl-2-thiazolyl)-2,5-diphenyl-2-H-tetrazolium bromide (MTT) assay at 1 and 2 days according to the manufacturer's specification and tested at at wave length of $570 \mathrm{~nm}$.

\subsection{Osteogenic gene expression}

For osteogenic related gene expression analysis, real time-quantitative polymerase chain reaction (RT-qPCR) was performed, briefly two types of scaffolds was produced and sterilized before using, then MC3T3-E1 cells were seeded onto scaffolds in the 6 -well culture plates at a density of $8 \times 10^{6}$ cells per well. MC3T3-E1 cells were incubated with steogenic differentiation medium $(\alpha-$ MEM medium, $100 \mathrm{U} / \mathrm{ml}$ penicillin, $100 \mathrm{mg} / \mathrm{ml}$ streptomycin, 10nmol/L dexamethasone, 10mmol/L $\beta$-glycerolphosphate, $10 \mathrm{nmol} / \mathrm{L}$ vitamin $\mathrm{D}$ and $10 \%$ fetal bovine serum) for 7 days, then total RNA was obtained by using TRIzol reagent (Invitrogen) according to the standard protocol. Subsequent $1 \mu \mathrm{g}$ total RNA was used for reverse transcription in to cDNA with Reverse Transcription Kit (TOYOBO, Japan), Finally, qPCR analysis was conducted by SYBR assay(KAPA, USA), and relative expression of osteogenesis related genes were analyzed by the $2-\Delta \Delta C T$ method. The mRNA expression level was normalized to the reference gene $\beta$-Actin. The specific primers used for PCR amplification were as follows ( $F$, forward; R,reverse):

ß-Actin, F 5'-GTGACGTTGACATCCGTAAAGA -3'and R 5'-GCCGGACTCATCGTACTCC-3', Osteocalcin(OCN) F 5'-CTGACCTCACAGATCCCAAGC and R 5'-TGGTCTGATAGCTCGTCACAAG,Runtrelated transcription factor 2 (RUNX2) $\mathrm{F}$ 5'-GACTGTGGTTACCGTCATGGC and $\mathrm{R}$ 5'-ACTTGGTTTTTCATAACAGCGGA,A Lkaline Phosphatase(ALP) F 5'GCCTTACCAACTCTTTTGTGCC and R 5'-CACCCGAGTGGTAGTCACAAT,Type Icollagen (Col I)F 5'-TAAGGGTCCCCAATGGTGAGA and R 5'-GGGTCCCTCGACTCCTACAT.

\subsection{In vivo implanted assay of PTMC/HA and PTMC/PLA/HA scaffolds in femur defect}

SD rats (weighted around 450g) were obtained from the Experimental Animal Center of Tongji Medical College (Wuhan, China). All rats were fed in the Specific Pathogen Free (SPF) room with professional equipment and abundant sterile water and food. They were randomly separated into 3 groups $(6$ rats per group): Control, PTMC/HA group and PTMC/PLA/HA group. For model conducted, two types of scaffolds were produced by $3 \mathrm{D}$ printing with parameters as follows: $\mathrm{HA}$ content $=25 \%$, diameter $=4 \mathrm{~mm}$, height $=$ $6 \mathrm{~mm}$. All were rinsed and sterilized by ethylene oxide before the surgery. SD rats were anesthetized with $3 \%$ pentobarbital, then the femur was exposed and $4 \mathrm{~mm}$ defect was made at the external epicondyle of femur. We used the muscle to press the defect hole in the Control group, for the rest two scaffolds groups, two types of scaffolds were implanted into it. After the operation, all rats were injected with with penicillin solution for 3 days and fed for another 2 months. Subsequently, the rats were performed with euthanasia, and the femurs were fixed with $4 \%$ paraformaldehyde. For evaluation of the defect reparation and new bone formation in the scaffolds, micro-computed tomography ( $\mu$-CT, Scanco Medical) scanning was performed, the scanning parameters were set as $100 \mathrm{kV}, 98 \mu \mathrm{A}$ and voxel size $=10 \mu \mathrm{m}$. Fracture surface and threedimensional images were obtained by using software built-in the Micro CT Scanner, the cylindrical area based on the defect area were calculated for new bone formation, the main parameters including trabecular numbers (Tb.N), trabecular space (Tb.Sp), bone volume/tissue volume (BV/TV) and trabecular thickness (Tb.Th) were analyzed.

\subsection{Statistical analysis}

For statistical analysis, all experiments were conducted independently [?]3 times, Images represent typical results of experiments and the results are revealed as the means \pm SD. Student's t tests method are used to analyze between two groups, and multiple comparisons are performed among more than two groups. P value less than 0.05 is regarded as significant difference. 


\section{Results and Discussions}

\subsection{Characterization of PTMC/HA and PTMC/PLA/HA scaffolds}

The biodegradable PTMC/HA and PTMC/PLA/HA scaffolds were produced by the blending and further 3D printing of composite materials based on PTMC, PLA and HA. In the PTMC/PLA/HA scaffolds, PTMC was chosen as a soft polymer segment whilst PLA showed a rigid polymer segment and HA was used as a reinforcing filler. PTMC/PLA/HA composite material was prepared by the modification of PTMC by PLA and HA and expected to improve the mechanical strength and mechanical property while maintaining flexibility. Moreover, the alkalescent HA nanoparticles could neutralize the acidic matrix of PLA and suppress the degradation process of ester bonds further.

The PTMC/HA and PTMC/PLA/HA scaffolds were characterized by Fourier transform infrared spectroscopy (FT-IR), automatic contact-angle, DSC, TG, XRD, SEM and mechanic properties. As shown in Figure. $\mathbf{1}$, the chemical characterization of pure HA, PTMC and two types of scaffolds printed by PTMC/HA and PTMC/PLA/HA (containing various content of HA) were performed by using the FT-IR analysis. When these substrates were mixed together, many of the absorption bands were over-lapped and the capability of functional groups staid the same. But the location, the shape and peak strengths changed greatly. The spectra of PTMC/HA scaffolds reflected a typical CH peak $(2923 \mathrm{~cm}-1)$ and ester peaks (respectively at $1748,1175,1030 \mathrm{~cm}^{-1}$ ), and accompanied by increased HA content percentage in scaffolds ranges from $0 \%$ to $25 \%$, it indicated a corresponding decrease of peak intensities. Moreover, PTMC/HA scaffolds revealed spectral features at 1080, 960, 603 and $564 \mathrm{~cm}^{-1}$, which reflected the vibrations of $\mathrm{P}=\mathrm{O}$ and $\mathrm{P}-\mathrm{O}$ in $\mathrm{HA}$, and ranging from 3500 to $3200 \mathrm{~cm}^{-1}$, which indicated the $\mathrm{OH}$ groups' absorption peak in the HA and PTMC, however, their peak intensities decreased greatly (Figure. 1a). Meanwhile, the spectra of PTMC/PLA/HA scaffolds showed typical $\mathrm{CH}$ peaks $\left(2963 \mathrm{~cm}^{-1}\right.$ ) and ester peaks (at 1948, 1181, $1060 \mathrm{~cm}^{-1}$ ), and accompanied by increased HA content percentage in scaffolds ranges from $0 \%$ to $25 \%$, the peak intensities were also decreased. Moreover, PTMC/PLA/HA scaffolds showed typical spectral features at 1080, 960, 615 and 564 $\mathrm{cm}^{-1}$, which showed the vibrations of $\mathrm{P}=\mathrm{O}$ and $\mathrm{P}-\mathrm{O}$ in $\mathrm{HA}$, and ranging from 3500 to $3200 \mathrm{~cm}^{-1}$, which revealed the $\mathrm{OH}$ groups' absorption peak in the HA and PTMC, and their peak intensities was declined greatly (Figure. 1b )

Water contact angles of PTMC/HA and PTMC/PLA/HA scaffolds were demonstrated in Figure. 1. Water contact angles of PTMC/HA decreased along with the increase of HA content from 0 to $20 \%$ and then increased when HA content percentage continuously increased from $20 \%$ to $25 \%$ (Figure. 1g ). The water contact angle of PTMC/HA scaffolds with $20 \% \mathrm{HA}$ content displayed the lowest water contact angles of $84.46^{\circ}$. Water contact angles of PTMC/PLA/HA decreased along with the increase of HA content from 0 to $15 \%$ and then increased when HA content percentage continuously increased from $15 \%$ to $25 \%$ (Figure. $\mathbf{1 h}$ ). The water contact angle of PTMC/PLA/HA scaffolds with $15 \%$ HA content displayed the lowest water contact angles of $56.80^{\circ}$. Moreover, PTMC/PLA/HA scaffolds possessed the lower water contact angles and higher hydrophilicity than that of PTMC/HA scaffolds. Therefore, the modification of PLA and HA to PTMC can enhanced obviously the hydrophilicity of composite materials.

Thermo property of PTMC/HA and PTMC/PLA/HA scaffolds were measured by DSC and TG (Figure. 1cdef and Table 1, 2 ).Figure. 1cd indicated the DSC curves of PTMC, PTMC/HA and PTMC/PLA/HA composite materials. PTMC/HA indicated the typical peaks of glass-transition temperature $(\mathrm{Tg})$ ranging from $-18^{\circ} \mathrm{C}$ to $-16^{\circ} \mathrm{C}$ and $\mathrm{Tg}$ of pure PTMC was $-16.7^{\circ} \mathrm{C}$, indicating HA had good compatibility to PTMC. It can be seen from Figure. 1c that the temperature of melting peak of PTMC was shifted by the addition of HA, which indicated that HA has some influence on the crystalline integrity and grain size of PTMC materials. On the one hand, with the increase of HA content, the damage of PTMC crystal particles and the decrease of crystal confinement and crystalline integrity were caused. On the other hand, the hydrogenbonding interaction of hydroxyl groups on the surface of HA with PTMC increased. Therefore, the melting peak temperature of PTMC/HA composites decreased gradually along with the increase of HA content from 0 to $10 \%$, reaching the minimum when the content of HA was $10 \%$. Subsequently, the melting peak temperature of the composites gradually increased again whilst the content of HA continued to increase from 
$10 \%$ to $25 \%$.

However, PTMC/PLA/HA revealed two typical glass-transition temperature (Tg) related peaks which ranged from $-14^{\circ} \mathrm{C}$ to $-12^{\circ} \mathrm{C}$ and varying from $46^{\circ} \mathrm{C}$ to $56^{\circ} \mathrm{C}$ whilst $\mathrm{Tg}$ of pure PTMC and PLA were $-16.7^{\circ} \mathrm{C}$ and $56^{\circ} \mathrm{C}$, respectively, indicating both PTMC and PLA existed in the composite materials. It can be seen from Figure. 1d that the temperature of melting peak of PTMC was shifted highly and the temperature of melting peak of PLA was moved lowly by the addition of HA, which indicated that HA has some influence on the crystalline integrity and grain size of PTMC and PLA materials. On the one hand, with the increase of HA content, the damage of PTMC and PLA crystal particles and the decrease of crystal confinement and crystalline integrity were caused. On the other hand, the hydrogen-bonding interaction of hydroxyl groups on the surface of HA with PTMC and PLA increased. Therefore, the melting peak temperature of PTMC component $\left(\mathrm{T}_{\mathrm{g} 1}\right)$ in PTMC/PLA/HA composites increased gradually along with the increased percentage of HA ranging from $0 \%$ to $15 \%$, reaching the maximum when the content of HA was $15 \%$. Subsequently, the melting peak temperature of PTMC component in the composites gradually decreased again whilst the content of HA continued to increase from $15 \%$ to $25 \%$. However, the melting peak temperature of PLA component $\left(\mathrm{T}_{\mathrm{g} 2}\right)$ in PTMC/PLA/HA composites decreased gradually along with the increased content of HA ranging from $0 \%$ to $15 \%$, reaching the minimum when the content of HA was $15 \%$. Subsequently, the melting peak temperature of PLA component in the composites gradually increased again whilst the content of HA continued to increase from $15 \%$ to $25 \%$. Moreover, the difference value of melting peak temperature $\left(\mathrm{T}_{\mathrm{g} 2}-\mathrm{T}_{\mathrm{g} 1}\right)$ of PTMC/PLA/HA composites decreased gradually along with the increased content of HA, reaching the minimum when the content of HA was $15 \%$. Subsequently, the difference value of melting peak temperature $\left(\mathrm{T}_{\mathrm{g} 2}-\mathrm{T}_{\mathrm{g} 1}\right)$ of the composites gradually increased again whilst the content of HA continued to increase from $15 \%$ to $25 \%$. These results demonstrated the addition of HA can promote the compatibility between PTMC and PLA.

Figure. 1ef and Table 2 show TG of pure PTMC, PTMC/HA and PTMC/PLA/HA composite materials, respectively, and then represent thermal degradation of PTMC,PTMC/HA and PTMC/PLA/HA. The effect of HA contents on the thermal properties of PTMC/HA and PTMC/PLA/HA scaffolds were analyzed. The thermal stability of PTMC was better than that of PTMC/HA composite materials. PTMC began to degrade at $258.6^{\circ} \mathrm{C}$ and ended at $307.9^{\circ} \mathrm{C}$, which indicated that PTMC had high thermal stability. For PTMC/HA composites, the addition of HA slightly reduced the thermal stability of the composites, and the thermal degradation curve moved to the low temperature zone. When the content of HA increased from $0 \%$ to $25 \%$, the degradation temperature for weight loss at $10 \%$ and $50 \%\left(\mathrm{~T}_{10 \%}\right.$ and $\left.\mathrm{T}_{50 \%}\right)$ showed a trend of decreasing gradually, and both degradation temperature for weight loss at $80 \%$ and maximum value $\left(\mathrm{T}_{80 \%}\right.$ and $\left.\mathrm{T}_{\max }\right)$ were in the state of increasing.

The thermal stability of PTMC/PLA/HA composites with lower HA content were better than that of PTMC/PLA composites, however, thermal stability of PTMC/PLA/HA composites decreased gradually with the increase of HA content. PTMC/PLA began to degrade at $279.4^{\circ} \mathrm{C}$ and ended at $386.3^{\circ} \mathrm{C}$, which indicated that PTMC/PLA had higher thermal stability than that of pure PTMC and PTMC/PLA/HA had higher thermal stability than that of PTMC/HA. For PTMC/PLA/HA composites, the addition of HA slightly decreased the composites' thermal stability, and the thermal degradation curve moved to the low temperature zone.

The degradation temperature accompanied by a weight loss at $10 \%, 50 \%, 80 \%$ and the maximum $\left(\mathrm{T}_{10 \%}\right.$, $\mathrm{T}_{50 \%}, \mathrm{~T}_{80 \%}$ and $\mathrm{T}_{\max }$ ) of PTMC/PLA/HA composites increased gradually along with the increased content of HA ranging from $0 \%$ to $15 \%$, reaching the maximum when the content of HA was $15 \%$. Subsequently, the degradation temperature with a weight loss of $10 \%, 50 \%, 80 \%$ and the maximum $\left(\mathrm{T}_{10 \%}, \mathrm{~T}_{50 \%}, \mathrm{~T}_{80 \%}\right.$ and $\mathrm{T}_{\max }$ ) of PTMC/PLA/HA composites gradually decreased again whilst the content of HA continued to increase from $15 \%$ to $25 \%$. These results also showed the addition of HA can promote the compatibility between PTMC and PLA.

\subsection{Scanning electronic micrographs of PTMC/HA and PTMC/PLA/HA scaffolds}


The micrographs of PTMC/HA and PTMC/PLA/HA scaffolds were examined by SEM. Figure. 2 shows that all PTMC/HA and PTMC/PLA/HA scaffolds presented the randomly morphology. HA nanoparticles scattered uniformity in the scaffolds and there were a few aggregated nanoparticles. The intensity of HA nanoparticles appeared increasingly according to the enlargement of HA content in scaffolds. The micrographs of PTMC/HA scaffolds showed that HA had good compatibility to PTMC. However, some hierarchical structures appear in the micrographs of PTMC/PLA/HA scaffolds, which indicating that both PTMC and PLA existed in the composite materials and the addition of HA can promote the compatibility between PTMC and PLA. These results are consistent with that of the above thermo properties of PTMC/HA and PTMC/PLA/HA scaffolds. Typical XRD spectra obtained for PTMC/HA and PTMC/PLA/HA scaffolds are presented in Figure. 2 d1, d2 . The spectra of PTMC/HA and PTMC/PLA/HA scaffolds also showed the same characteristic peaks as PTMC. 3D printed PTMC/HA and PTMC/PLA/HA scaffolds possessed the different uniform pore size structures.

The compressive strength and modulus of PTMC/HA and PTMC/PLA/HA scaffolds were measured and shown in Figure. 3a andTable $\mathbf{3 b}$. The compressive strength and modulus of PTMC/HA scaffolds increased along with the increased content of HA ranging from $0 \%$ to $15 \%$. HA was uniformly dispersed in the PTMC polymer matrix. When the composite is affected by external force, it blocked the movement of the molecular chain, thus increasing the compressive properties of the PTMC/HA composites. Moreover, PTMC/HA and PTMC/PLA/HA scaffolds possessed the obviously higher compressive strength and modulus than that of PTMC and PTMC/HA scaffolds, respectively. These results showed that the addition of HA can promote the compressive property of PTMC/HA. The compressive strength and modulus of PTMC/HA and PTMC/PLA/HA scaffolds increased gradually along with the increase of HA content from 0 to $5 \%$, reaching the maximum when the content of HA was $5 \%$. Subsequently, compressive strength and modulus of PTMC/PLA/HA composites gradually decreased again whilst the content of HA continued to increase from $5 \%$ to $25 \%$.

\subsection{In vitro degradation}

The in vitro degradation of PTMC/HA and PTMC/PLA/HA scaffolds were measured in PBS at $37^{\circ} \mathrm{C}$. During the degradation process in PBS for 6 months, PTMC/HA and PTMC/PLA/HA with 10\% and $25 \%$ HA content experienced weight loss and molecular weight loss increased with time. The weight losses of PTMC/HA scaffolds, PTMC/PLA/HA scaffolds with $10 \%$ HA content and PTMC/PLA/HA scaffolds containing $25 \% \mathrm{HA}$ were $8 \%, 12 \%$, and $32 \%$ at the end of 6 months, respectively (Figure. 4b ). PTMC/PLA/HA and PTMC/HA scaffolds containing $25 \%$ HA accordingly displayed the obviously higher weight loss than that of PTMC/PLA and PTMC scaffolds, respectively. Whilst PTMC/PLA/HA with 10\% HA content possessed the slightly higher weight loss than that of PTMC/PLA and PTMC scaffolds and the lower weight loss than that of PTMC/PLA/HA and PTMC/HA scaffolds containing $25 \% \mathrm{HA}$ accordingly.

On the other hand, the molecular weight of PTMC/PLA and PTMC/PLA/HA scaffolds containing $25 \%$ HA decreased significantly, while that of PTMC/PLA/HA scaffolds with $10 \%$ HA content was relatively little (Figure. 4d ). The degradation of PLA resulted in local $\mathrm{pH}$ reduction, which accelerated the degradation of the PTMC/PLA and PTMC/PLA/HA composites. So PTMC/PLA composite materials contained relatively more PLA and then PTMC/PLA scaffolds indicated high molecular weight loss after 6 months degradation. PTMC/PLA/HA scaffolds containing $25 \%$ HA contained the largest amount of HA, which maybe aggregate as nanoparticles and form the big large holes and cracks in the scaffolds after the removal of HA from the composites in the degradation process. Meanwhile, the increasing of water permeation further accelerated hydrolysis and degradation. Thus PTMC/PLA/HA scaffolds containing $25 \%$ HA had the highest molecular weight and also weight loss due to the high permeability and promoted degradation rate. PTMC/PLA/HA scaffolds with $10 \% \mathrm{HA}$ content had lower weight loss, molecular weight loss and degradation rate than PTMC/PLA/HA scaffolds containing 25\% HA since HA nanoparticles scattered uniformity in PTMC/PLA composite matrix. Because of the alkalescent HA nanoparticles, it could the neutralize acidic substances in PLA, and further suppressed the degradation process of ester bonds. These results indicated that hydroxyl groups of HA and pores in the matrix enhanced the hydrophilicity, water absorption and degradation rate 
of scaffolds.

\subsection{In vitro drug-release property of PTMC/HA and PTMC/PLA/HA scaffolds}

DOX was used as a typical model anticancer drug model for drug-release property of PTMC/HA and PTMC/PLA/HA scaffolds because of its easy to detect by HPLC and UV. The DOX release properties of pure PTMC, PTMC/HA and PTMC/PLA/HA scaffolds are shown in Figure. 5 . The substantial releases of the DOX-incorporated pure PTMC, PTMC/HA and PTMC/PLA/HA scaffolds were maintained for 65 days of measurement. The DOX-incorporated pure PTMC, PTMC/HA and PTMC/PLA/HA scaffolds displayed steady drug-release rates and good drug-controlled release properties.

Compared with DOX-incorporated pure PTMC scaffolds, DOX-incorporated PTMC/HA and PTMC/PLA/HA scaffolds possessed the faster drug-release rates, presumably due to the increased drug diffusion coefficient of PTMC/HA and PTMC/PLA/HA scaffolds. Moreover, the release rate increased along with the increasing of HA content. The cumulative percentage release of PTMC/HA scaffolds (with HA content: $10 \%$ and $25 \%$ ) reached $10.8 \%$ and $11.6 \%$, respectively, and higher than that of pure PTMC scaffolds (8.0\%), after 45 days of drug-release. The cumulative percentage release of PTMC/PLA/HA scaffolds (with HA content: $10 \%$ and $25 \%$ ) reached $7.3 \%$ and $8.1 \%$, respectively, and higher than that of PTMC/PLA scaffolds (6.4\%), after 55 days of drug-release. This result indicated that hydroxyl groups of $\mathrm{HA}$ and pores in the matrix enhanced the hydrophilicity, water absorption and drug diffusion coefficients of scaffolds.

\subsection{Cell cytotoxicity assay}

Metabolic activity of the osteoblast cells MC3T3-E1 cultured with pure PTMC, PTMC/HA and PTMC/PLA/HA scaffolds was evaluated by the CCK-8 assay on 1- and 3-days post culturing (Figure. 6 ). All scaffolds could support the growth of MC3T3-E1 cells. There were no significant differences of cell proliferation activity among all scaffolds at 1 day. The cell proliferation on PTMC/HA scaffolds with $15 \%$ HA content and $25 \%$ HA content were slightly higher than that of the other PTMC/HA scaffolds. The cell proliferation on PTMC/PLA/HA scaffolds with 5\% HA content and 10\% HA content were slightly higher than that of the other PTMC/PLA/HA scaffolds. Moreover, PTMC/PLA/HA scaffolds possessed lower cell cytotoxicity and higher cell proliferation than that of PTMC/HA scaffolds.

However, these scaffolds revealed a different cell proliferation capacity at 3 days. PTMC/HA scaffolds containing 25\% HA and pure PTMC had the higher cell proliferation activity than that of the other PTMC/HA scaffolds. PTMC/PLA scaffolds displayed lower cell proliferation activity than that of PTMC/PLA/HA scaffolds containing 25\% HA, meanwhile, higher than that of the other PTMC/PLA/HA scaffolds with $5 \%, 10 \%, 15 \%$ and 20\% HA content. Moreover, PTMC/PLA/HA scaffolds also possessed lower cell cytotoxicity and higher cell proliferation than that of PTMC/HA scaffolds. These results demonstrated that PTMC/PLA/HA scaffolds and PTMC/HA scaffolds containing 25\% HA indicated good biocompatibility and showed a good biological ability for cell proliferation.

According to the analysis of experimental results of cell cytotoxicity assay, FT-IR, automatic contact-angle, DSC, TG, XRD, SEM and compressive modulus above, PTMC/HA and PTMC/PLA/HA scaffolds 25\% HA content (PTMC/25wt\%HA and PTMC/PLA/25wt\%HA) possessed the highest cell proliferation performances and were chosen as the samples to further investigate the cell viability in vitro and proliferation and new bone regeneration with femur defect model in vivo .Figure. 6 shows the pictures of 3D printing composite scaffold with obvious network structure produced by $3 \mathrm{D}$ printing technology. Through the test of shrinkage and porosity of the scaffold, 3D printed PTMC/25wt\%HA scaffolds showed the porosity of 65 +- $0.82 \%$ and shrinkage of $15.6+-0.36 \%$, whilst 3D printed PTMC/PLA/25wt\%HA scaffolds displayed the porosity of $67+-0.82 \%$ and shrinkage of $15.8+-0.29 \%$.

\subsection{In vitro cell studies of PTMC/25wt\%HA and PTMC/PLA/25wt\%HA scaffolds}

Metabolic activity of the osteoblast cells MC3T3-E1 cultured with the PTMC/25wt\%HA and PTMC/PLA/25wt\%HA scaffolds were evaluated by the 3-(4,5-dimethyl-2-thiazolyl)-2,5-diphenyl-2-H- 
tetrazolium bromide (MTT) assay, real time fluorescent quantitative PCR and Western blot assay on 7 days postculturing. The expression of $\beta$-Actin, collagen (COL), Osteocalcin (OCN), Alkaline phosphatase (ALP) and Runt related transcription factor-2 (RUNX2) at mRNA level were performed by using qPCR and revealed in Figure. 7c. As showed in Figure 7 a-c, PTMC/PLA/25wt\%HA scaffolds displayed higher cell adhesion capacity and proliferation activity than that of PTMC/25wt\%HA scaffolds by the phalloidin staining and MTT assay.

PTMC/25wt\%HA and PTMC/PLA/25wt\%HA scaffolds supported the growth of MC3T3-E1 cells and obviously promoted the expression of COL I, OCN, ALP and RUNX2 at mRNA level. Moreover, MC3T3-E1 cells scattered uniformity and displayed the good adhesion and cell proliferation activity on the surface of 3D branches in PTMC/25wt\%HA and PTMC/PLA/25wt\%HA scaffolds (Figure. 7c ). PTMC/25wt\%HA and PTMC/PLA/25wt\%HA scaffolds evidently promoted the expression of COL I, OCN, ALP and RUNX2. These osteogenesis related genes induced by PTMC/25wt\%HA scaffolds indicated a higher expression level than that of PTMC/PLA/25wt\%HA scaffolds. These results demonstrated that both two typed of scaffolds revealed a good biocompatibility, and PTMC/25wt\%HA scaffolds showed a good capacity at osteogenesis.

\subsection{PTMC/25wt\%HA/ PTMC/PLA/25wt\%HA scaffolds promoted the bone reparation in} femur defect model

Based on the in vitro consequences that PTMC/25wt\%HA/ PTMC/PLA/25wt\%HA scaffolds had an excellent physical and biological ability, SD rats were conducted with femur defect, after the anesthesia, a bone defect (diameter $=4 \mathrm{~mm}$, the height $=6 \mathrm{~mm}$ ) were performed at the external tuberosity of femur according the method reported as previous study ${ }^{29,30}$. Then SD rats of the Control groups were pressed with the muscle and sutured, the PTMC/25wt\%HA/ PTMC/PLA/25wt\%HA scaffolds groups were implanted the 3D printed and sterile scaffolds. After 2 months feeding, the femurs were fixed with with $4 \%$ paraformaldehyde and $\mu$-CT scanning was performed, the cylindrical area based on the defect area were calculated for new bone formation. Figure. 8a revealed the good biological capacity of PTMC/25wt\%HA and PTMC/PLA/25wt\%HA scaffolds, which indicated among the porosity and the branches, abundant new bone formation appeared and repaired the bone defect. Meanwhile, the PTMC/25wt\%HA and PTMC/PLA/25wt\%HA scaffolds were slowly biodegraded.

Compared with the PTMC/PLA/25wt\%HA scaffolds group, the PTMC/25wt\%HA scaffolds showed more new bone tissue and the data analysis indicated a higher bone tissue volume/total tissue volume (BV/TV), larger trabecular thickness (Tb.Th), higher trabecular space (Tb.Sp) and less number of trabecular (Tb.N). Probably the prolonged acidic byproducts of PLA degradation played negative effects on the cell proliferation and bone reparation.

\section{Conclusions}

The PTMC/HA and PTMC/PLA/HA scaffolds were prepared by 3D printing of composite materials based on PTMC, PLA and HA and possessed good biodegradability, good biocompatibility, and improved cell proliferation of MC3T3-E1. Moreover, PTMC/HA and PTMC/PLA/HA scaffolds could provide a appropriate microenvironment for osteogenesis and then display the remarkable osteogenic activity. Therefore, PTMC/HA and PTMC/PLA/HA composite materials enable the proliferation of bone cells in vitro and bone tissue regeneration in vivo, these materials could be potential biomaterials for bone repatriation and tissue engineering.

\section{Compliance with Ethical Standards}

In this study, the animal experiments were conducted at Experimental Animal Center of Tongji Hospital. All rats were raised in the professional cages with the 12-hour light/dark cycle, the air renewal system and constant temperature around 25. The animal experiments were approved by the Ethics Committee of Tongji Hospital, Tongji medical college, Huazhong University of Science and Technology.

\section{Acknowledgements}


This study was supported by grants from National Key R\&D Program of China (No. 2018YFB1105502, 2016YFB1101302), The Yellow Crane Talent Program of Wuhan City (Grant [2017] No.1), Frontier Project of Application Foundation of Wuhan Former Funded Science and Technology Program (Grant No. 2020020601012252), Hubei Province Key Research and Development Program (2020BCB049), Scientific research projects for high level talents in the new century of Hubei Province (Grant [2017] No.344) and South Hubei Talents Project of Innovation and Entrepreneurship (Grant [2019] No.11).

\section{Declaration of Competing Interest}

There are no conflicts of interest to declare.

\section{References:}

1. Matai I, Kaur G, Seyedsalehi A, McClinton A, Laurencin CT. Progress in 3D bioprinting technology for tissue/organ regenerative engineering.Biomaterials. 2020;226:119536.

2. Wang K, Ho C-C, Zhang C, Wang B. A Review on the 3D Printing of Functional Structures for Medical Phantoms and Regenerated Tissue and Organ Applications. Engineering. 2017;3(5):653-662.

3. Mandrycky C, Wang Z, Kim K, Kim DH. 3D bioprinting for engineering complex tissues. Biotechnol Adv. 2016;34(4):422-434.

4. Wang X, Zhang M, Ma J, et al. 3D Printing of Cell-Container-Like Scaffolds for Multicell Tissue Engineering. Engineering.2020;6(11):1276-1284.

5. Yan Y, Chen H, Zhang H, et al. Vascularized 3D printed scaffolds for promoting bone regeneration. Biomaterials. 2019;190-191:97-110.

6. Bunpetch V, Zhang X, Li T, et al. Silicate-based bioceramic scaffolds for dual-lineage regeneration of osteochondral defect.Biomaterials. 2019;192:323-333.

7. Zhuang P, Sun AX, An J, Chua CK, Chew SY. 3D neural tissue models: From spheroids to bioprinting. Biomaterials. 2018;154:113-133.

8. Daly AC, Pitacco P, Nulty J, Cunniffe GM, Kelly DJ. 3D printed microchannel networks to direct vascularisation during endochondral bone repair. Biomaterials. 2018;162:34-46.

9. Wang X, Jiang M, Zhou Z, Gou J, Hui D. 3D printing of polymer matrix composites: A review and prospective. Composites Part B: Engineering. 2017;110:442-458.

10. Jang J, Park JY, Gao G, Cho DW. Biomaterials-based 3D cell printing for next-generation therapeutics and diagnostics. Biomaterials.2018;156:88-106.

11. Liu D, Nie W, Li D, et al. 3D printed PCL/SrHA scaffold for enhanced bone regeneration. Chemical Engineering Journal.2019;362:269-279.

12. Guney A, Malda J, Dhert WJA, Grijpma DW. Triblock copolymers based on epsilon-caprolactone and trimethylene carbonate for the 3D printing of tissue engineering scaffolds. Int J Artif Organs.2017;40(4):176184.

13. Ligon SC, Liska R, Stampfl J, Gurr M, Mulhaupt R. Polymers for 3D Printing and Customized Additive Manufacturing. Chem Rev.2017;117(15):10212-10290.

14. Hu B, Yan G-P, Zhuo R-X, Wu Y, Fan C-L. Polycarbonate microspheres containing tumor necrosis factor- $\alpha$ genes and magnetic powder as potential cancer therapeutics. Journal of Applied Polymer Science. 2008;107(5):3343-3349.

15. Hu B, Du HJ, Yan GP, Zhuo RX, Wu Y, Fan CL. Magnetic polycarbonate microspheres for tumortargeted delivery of tumor necrosis factor.Drug Deliv. 2014;21(3):204-212. 
16. Feng TJ, Mei LL, Liu F, Yan GP, Yuan Y, Guo QZ. Microwave-assisted ring-opening copolymerization and property of polycarbonates.Polymers for Advanced Technologies. 2021;32(9):3412-3420.

17. Esposito Corcione C, Gervaso F, Scalera F, et al. Highly loaded hydroxyapatite microsphere/ PLA porous scaffolds obtained by fused deposition modelling. Ceramics International.2019;45(2):2803-2810.

18. Han SH, Cha M, Jin YZ, Lee KM, Lee JH. BMP-2 and hMSC dual delivery onto 3D printed PLA-Biogel scaffold for critical-size bone defect regeneration in rabbit tibia. Biomed Mater. 2020;16(1):015019.

19. Alam F, Shukla VR, Varadarajan KM, Kumar S. Microarchitected 3D printed polylactic acid (PLA) nanocomposite scaffolds for biomedical applications. J Mech Behav Biomed Mater. 2020;103:103576.

20. Huang KH, Lin YH, Shie MY, Lin CP. Effects of bone morphogenic protein-2 loaded on the 3D-printed MesoCS scaffolds. J Formos Med Assoc. 2018;117(10):879-887.

21. Chen L, Deng C, Li J, et al. 3D printing of a lithium-calcium-silicate crystal bioscaffold with dual bioactivities for osteochondral interface reconstruction. Biomaterials.2019;196:138-150.

22. Ramirez-Agudelo R, Scheuermann K, Gala-Garcia A, et al. Hybrid nanofibers based on polycaprolactone/gelatin/hydroxyapatite nanoparticles-loaded Doxycycline: Effective anti-tumoral and antibacterial activity. Mater Sci Eng C Mater Biol Appl.2018;83:25-34.

23. Ma H, Feng C, Chang J, Wu C. 3D-printed bioceramic scaffolds: From bone tissue engineering to tumor therapy. Acta Biomater.2018;79:37-59.

24. Li D, Zhang K, Shi C, et al. Small molecules modified biomimetic gelatin/hydroxyapatite nanofibers constructing an ideal osteogenic microenvironment with significantly enhanced cranial bone formation.Int $J$ Nanomedicine. 2018;13:7167-7181.

25. Chen H, Yan G-P, Li L, Ai C-W, Yu X-H. Synthesis, characterization, and properties of $\varepsilon$-caprolactone and carbonate copolymers. Journal of Applied Polymer Science. 2009;114(5):3087-3096.

26. Liao LQ, Liu LJ, Zhang C, He F, Zhuo RX, Wan K. Microwave-assisted ring-opening polymerization of ?-caprolactone. Journal of Polymer Science Part A: Polymer Chemistry. 2002;40(11):1749-1755.

27. Liu F, Mei L-l, Tan Z-l, et al. Studies on microwave-assisted ring-opening polymerization and property of poly(9-phenyl-2,4,8,10-tetraoxaspiro-[5,5] undcane-3-one). Chinese Journal of Polymer Science. 2016;34(11):1330-1338.

28. Wu X, Liao Z, Wang K, et al. Targeting the IL-1beta/IL-1Ra pathways for the aggregation of human islet amyloid polypeptide in an ex vivo organ culture system of the intervertebral disc. Exp Mol Med.2019;51(9):116.

29. Liu Y, Li T, Ma H, et al. 3D-printed scaffolds with bioactive elements-induced photothermal effect for bone tumor therapy. Acta Biomater. 2018;73:531-546.

30. Feng P, Wu P, Gao C, et al. A Multimaterial Scaffold With Tunable Properties: Toward Bone Tissue Repair. Adv Sci (Weinh).2018;5(6):1700817.

Table 1 DSC data of PTMC/HA and PTMC/PLA/HA scaffolds

\begin{tabular}{llll}
\hline & $\mathrm{T}_{\mathrm{g} 1}()$ & $\mathrm{T}_{\mathrm{g} 2}()$ & $\mathrm{T}_{\mathrm{g} 2}-\mathrm{T}_{\mathrm{g} 1}()$ \\
\hline PTMC & -16.7 & & \\
PTMC/5\%HA & -17.0 & & \\
PTMC/10\%HA & -17.5 & & \\
PTMC/15\%HA & -16.9 & & \\
PTMC/20\%HA & -16.7 & & \\
PTMC/25\%HA & -16.2 & &
\end{tabular}




\begin{tabular}{llll}
\hline & $\mathrm{T}_{\mathrm{g} 1}()$ & $\mathrm{T}_{\mathrm{g} 2}()$ & $\mathrm{T}_{\mathrm{g} 2}-\mathrm{T}_{\mathrm{g} 1}()$ \\
\hline PTMC/PLA & -13.9 & 54.9 & 68.8 \\
PTMC/PLA/5\%HA & -12.9 & 55.6 & 68.5 \\
PTMC/PLA/10\%HA & -13.0 & 55.0 & 68.0 \\
PTMC/PLA/15\%HA & -12.2 & 46.6 & 58.9 \\
PTMC/PLA/20\%HA & -13.0 & 54.9 & 67.9 \\
PTMC/PLA/25\%HA & -15.6 & 55.0 & 70.6 \\
\hline
\end{tabular}

Table 2 Thermo property of PTMC/HA and PTMC/PLA/HA scaffolds

\begin{tabular}{lllllll}
\hline Heating rate $(/ \min )$ & $\mathrm{T}_{10 \%}()$ & $\mathrm{T}_{50 \%}()$ & $\mathrm{T}_{80 \%}()$ & $\mathrm{T}_{\max }()$ & Residue at $400(\%)$ & Residue at $450(\%)$ \\
\hline PTMC & 258.6 & 280.6 & 291.7 & 307.9 & 0.3 & 0.3 \\
PTMC/5\%HA & 227.1 & 274.1 & 292.5 & 329.6 & 8.6 & 8.3 \\
PTMC/10\%HA & 230.1 & 278.4 & 297.7 & 331.2 & 9.3 & 9.1 \\
PTMC/15\%HA & 223.2 & 268.9 & 287.6 & 337.2 & 12.4 & 12.3 \\
PTMC/20\%HA & 227.8 & 280.1 & 304.1 & 342.1 & 15.7 & 15.4 \\
PTMC/25\%HA & 224.8 & 277.9 & 317.9 & 349.5 & 19.2 & 0.4 \\
PTMC/PLA & 298.9 & 317.0 & 328.1 & 376.3 & 0.7 & 6.0 \\
PTMC/PLA/5\%HA & 302.5 & 335.8 & 347.1 & 380.8 & 6.3 & 5.3 \\
PTMC/PLA/10\%HA & 307.1 & 335.4 & 347.9 & 378.0 & 5.8 & 15.8 \\
PTMC/PLA/15\%HA & 267.1 & 333.0 & 352.9 & 395.1 & 16.5 & 19.3 \\
PTMC/PLA/20\%HA & 246.5 & 311.0 & 365.0 & 362.6 & 19.6 & 25.9 \\
PTMC/PLA/25\%HA & 231.7 & 293.0 & - & 340.1 & 26.0 & \\
\hline
\end{tabular}

Table 3 Compressive strength and modulus of PTMC/HA and PTMC/PLA/HA scaffolds

\begin{tabular}{lll}
\hline & Compressive modulus $(\mathrm{MPa})$ & $\mathrm{F}_{\sigma}=50 \%(\mathrm{~N})$ \\
\hline PTMC & 1.54 & 80.64 \\
PTMC/5\%HA & 4.61 & 220.96 \\
PTMC/10\%HA & 5.05 & 254.20 \\
PTMC/15\%HA & 6.44 & 296.41 \\
PTMC/20\%HA & 6.75 & 307.43 \\
PTMC/25\%HA & 8.07 & 325.89 \\
PTMC/PLA & 348.34 & 3057 \\
PTMC/PLA/5\%HA & 409.87 & 3513 \\
PTMC/PLA/10\%HA & 381.22 & 3236 \\
PTMC/PLA/15\%HA & 328.94 & 2961 \\
PTMC/PLA/20\%HA & 320.89 & 2932 \\
PTMC/PLA/25\%HA & 182.48 & 2019 \\
\hline
\end{tabular}

\section{Figure Legends}

Figure 1. FT-IR (a), DSC (c), TG (e), and water contact angle (g) of PTMC/HA; FT-IR (b), DSC (d), TG (f), and water contact angle (h) of PTMC/PLA/HA scaffolds

Figure 2. Micromorphology of PTMC/HA and PTMC/PLA/HA scaffolds

(a1: PTMC, b1: PTMC/10\%HA, c1: PTMC/25\%HA, a2: PTMC/PLA, b2: PTMC/PLA/10\%HA, c2: 
PTMC/PLA/25\%HA); XRD of PTMC/HA (d1) and PTMC/PLA/HA scaffolds (d2)

Figure 3. Compressive modulus of PTMC/HA (a) and PTMC/PLA/HA (b) scaffolds

Figure 4. Weight loss and number average molecular weight loss of PTMC/PLA and PTMC/PLA/HA scaffolds

Figure 5. Release profiles of DOX from PTMC/HA (a) and PTMC/PLA/HA (b) scaffolds

Figure 6. Cell proliferation assays of the osteoblast cells MC3T3-E1. (a)PTMC/25wt\%HA scaffolds, (b) PTMC/PLA/25wt\%HA scaffolds.

Figure 7. Phalloidin staining images of cells after 7 days culturing on the scaffolds of (a) PTMC/25wt\%HA scaffolds, and (b) PTMC/PLA/25wt\%HA scaffolds. (c) MTT assay and Effects of the scaffolds on mRNA expression of the Actin, Runx2, ALP and Col I genes on day 7.

Figure 8. Micro-CT analysis of the effect of scaffolds on femur defect in vivo .

(a) Representative three-dimensional reconstructed micro-CT images showing the effect of PTMC $/ 25 \mathrm{wt} \% \mathrm{HA}$ and PTMC/PLA/25wt\%HA scaffolds on the new bone tissue formation inside the defect site (red dashed line). (Left: PTMC/25wt\%HA scaffolds for 2 months, right: PTMC/PLA/25wt\%HA scaffolds for 2 months); (b) Summarized data showing the micro-architectural parameters of the new formed bone tissue at 2 months by analyzing the three-dimensional reconstructed micro-CT images using image analysis software. BMD, $\mathrm{BV} / \mathrm{TV}$, Tb.Th and Tb.N were shown in the panel.
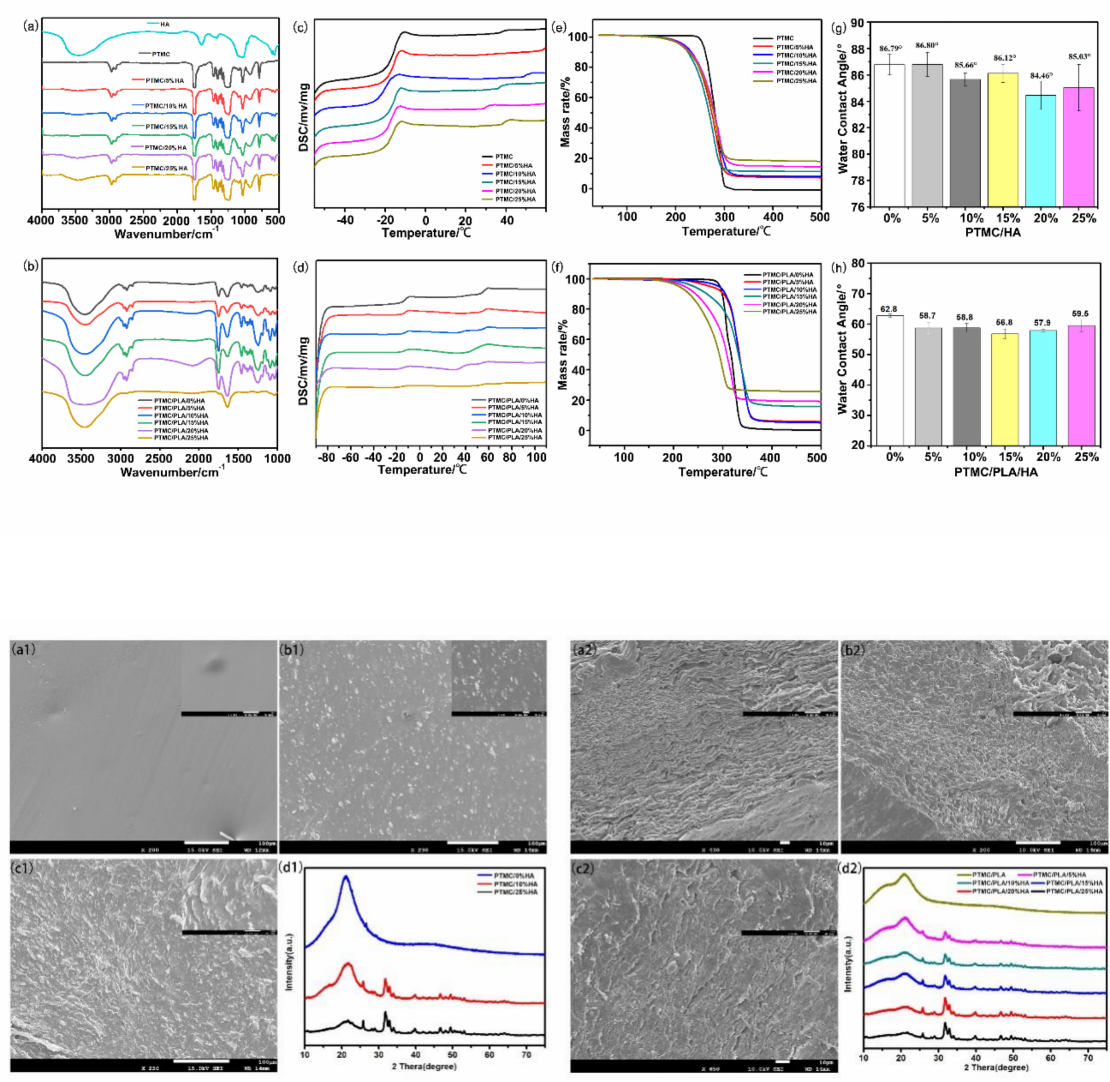

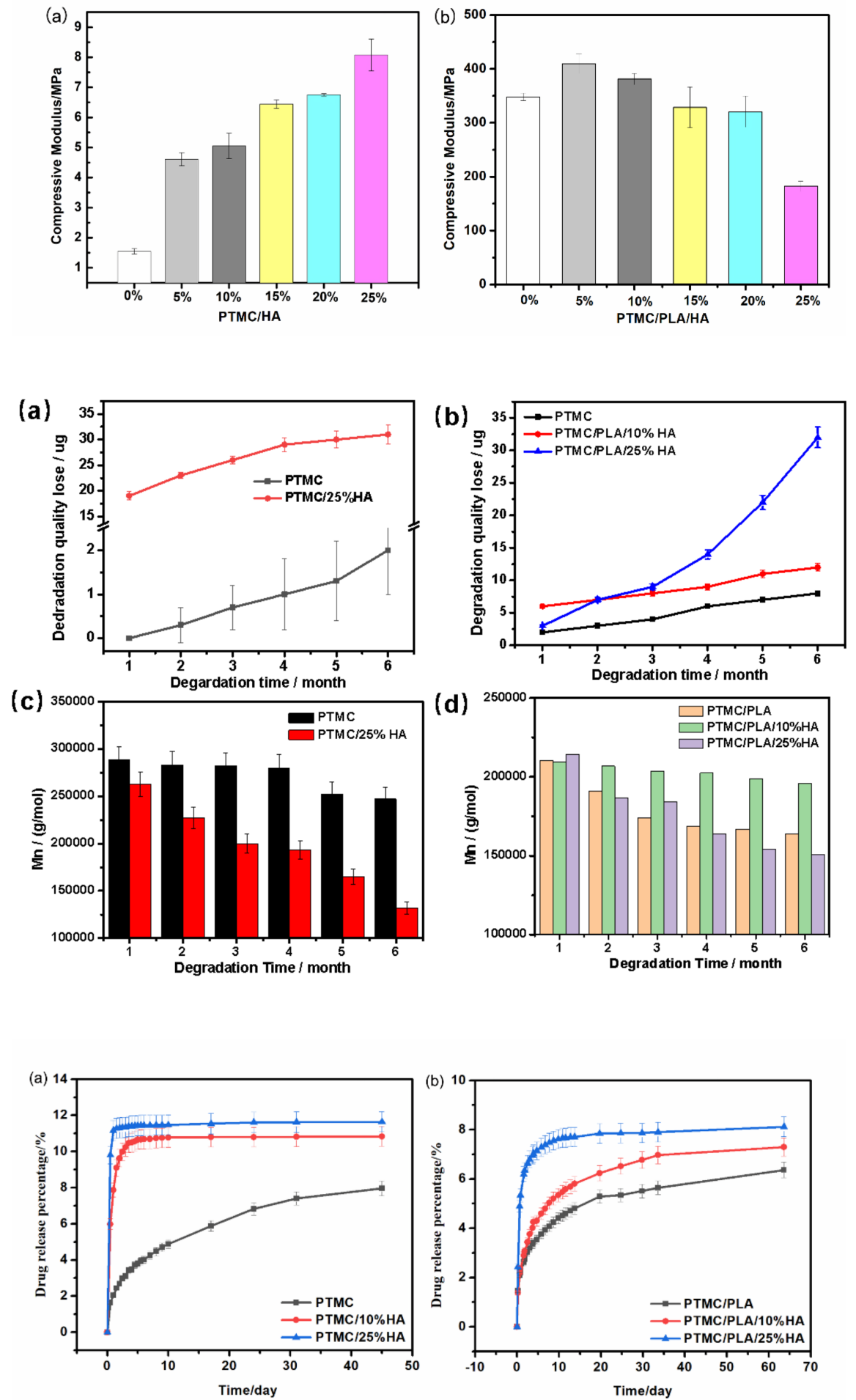

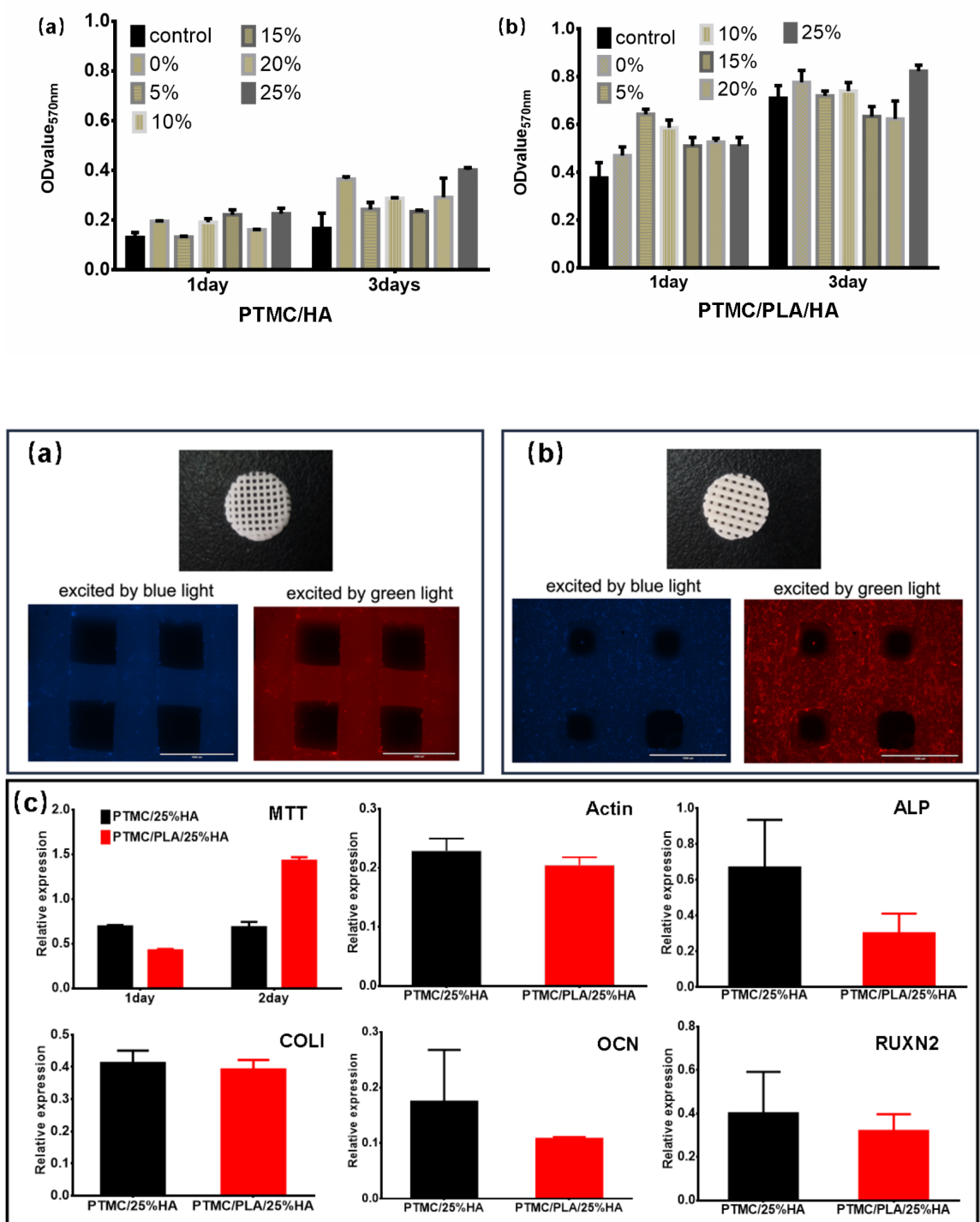


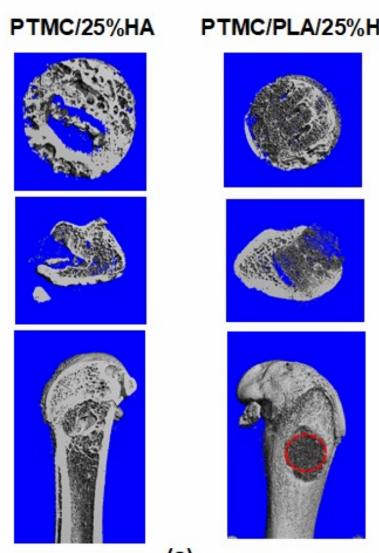

(a)

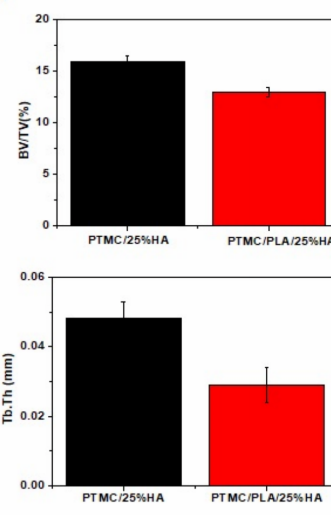

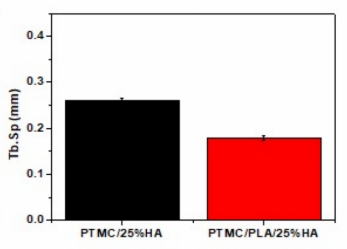

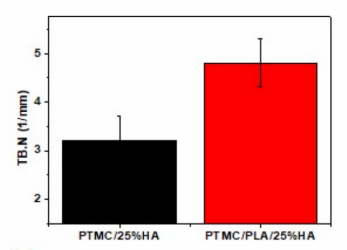

(b) 